Pacific Journal of Mathematic 


\section{GENERALIZED RIGHT ALTERNATIVE RINGS}

\section{IRVIN ROY HENTZEL}

We show that weakening the hypotheses of right alternative rings to the three identities

(1) $(a b, c, d)+(a, b,[c, d])=a(b, c, d)+(a, c, d) b$

(2) $(a, a, a)=0$

(3) $([a, b], b, b)=0$

for all $a, b, c, d$ in the ring will not lead to any new simple rings. In fact, the ideal generated by each associator of the form $(a, b, b)$ is a nilpotent ideal of index at most three. Our proofs require characteristic $\neq 2, \neq 3$.

Introduction. We shall call a ring a GRA ring (for generalized right alternative ring) if it satisfies the following three identities:

(1) $0 \equiv \bar{A}(a, b, c, d)=(a b, c, d)+(a, b,[c, d])-a(b, c, d)-(a, c, d) b$

(2) $0 \equiv(a, a, a)$

(3) $0 \equiv([b, a], a, a)$.

On all rings that we study in this paper, we assume that for $n=2$ or $n=3$, the map $x \rightarrow n x$ is one-to-one and onto. This is equivalent to weakly characteristic $\neq 2, \neq 3$ (see [1]). All three conditions are consequences of the right alternative law $(a, x, x) \equiv 0$ and characteristic $\neq 2$, and thus GRA rings are generalizations of right alternative rings. Similar conditions have been studied by E. Kleinfeld, H. F. Smith, I. R. Hentzel, and G. M. Piacentini, usually through an idempotent decomposition. The results given here generalize much of their work, mainly by dispensing with the assumption of an idempotent. Our work shows the relationship of these rings to right alternative rings; this is a simpler and more direct approach than that which has been done before.

When we are dealing with a GRA ring $R$, we shall let $I$ be the additive subgroup generated by all associators of the form $(a, b, b)$ for all $a, b \in R$. I is a measure of how far $R$ is from being a right alternative ring. We show that $I$ is an ideal of $R$, that $I$ is commutative, and that $I$ is the sum of ideals of $R$ whose cube is zero. This means that if $R$ is simple, or even nil-semi-simple, then $R$ is right alternative. Since all the 
hypotheses on $R$ are consequences of the right alternative law, showing that $R$ is right alternative is as strong a result as one could hope for.

The three hypotheses chosen are individually expressive of wellknown ring structure. Given (1), then (2) holds $\Leftrightarrow$ the ring is powerassociative. Given (1) and (2), then (3) holds $\Leftrightarrow$ the ring under the symmetric product $a \circ b=a b+b a$ is a Jordan ring.

Basic identities and definitions. An ideal $I$ of $R$ is called trivial if $I \neq 0$ and $I^{2}=0$. A ring $R$ is called semi-prime if $R$ has no trivial ideals. $\quad R$ is called simple if $R^{2} \neq 0$ and $R$ has no ideals except 0 and $R$ itself. The associator $(a, b, c)$ is defined by $(a, b, c)=$ $(a b) c-a(b c)$. The commutator $[a, b]$ is defined by $[a, b]=a b-b a$.

To simplify the notation, dot and juxtaposition will be used to indicate multiplication. When both appear, juxtaposition indicates that product is taken first. Thus $a b \cdot c=(a b) c$.

In expressions where elements are supposed to appear, we often place a set of elements. This means we are considering the additive group spanned by all the elements generated as the arguments of the expression vary through the indicated sets. Thus $(R, x, x)$ means the additive subgroup generated by $\{(r, x, x) \mid r \in R\}$.

The following identities are used:

(5) $0 \equiv \bar{C}(a, b, c)=[a,(b, c, c)]+[c,(b, a, c)]+[c,(b, c, a)]$.

(6) $0 \equiv \bar{D}(a, b, c, d)=(a, b, c d)-(a, b d, c)-(a, b, d) c+(a, d, c) b$.

(7) $0 \equiv \bar{E}(a, b, c, d)=(a b, c, d)-(a, b c, d)+(a, b, c d)$

$$
-a(b, c, d)-(a, b, c) d \text {. }
$$

Proof. Property (4) is a linearization of property (2). To show (5), it will suffice to show $0 \equiv[a,(b, a, a)]$. This follows since $0 \equiv$ $([b, a], a, a)+\bar{A}(a, b, a, a)-\bar{A}(b, a, a, a)=-[a,(b, a, a)]$. Property (7) is the Teichmüller equality which holds in any non-associative ring. Property (6) follows since $\bar{A}(a, b, d, c)+\bar{D}(a, b, c, d)=\bar{E}(a, b, d, c)$.

Main section. For comparison with other papers discussed in the final section of this paper, we will need a form of Lemma 1 and Lemma 2 that does not require (3).

LEMMA 1. Let $R$ be a nonassociative ring satisfying (1) and (2). Then $0 \equiv[a,(b, a, a)]+4(b, a, a) a-2\left(b, a, a^{2}\right)$. 
Proof.

$$
\begin{aligned}
0= & \bar{A}(a, a, a, b)+\bar{A}(a, a, b, a)-\bar{A}(b, a, a, a)+\bar{D}(a, b, a, a) \\
& -\bar{D}(a, a, b, a)-\bar{D}(b, a, a, a)+a \cdot \bar{B}(b, a)+2 \bar{B}(b, a) \cdot a+\bar{B}(b a, a) \\
= & a(b, a, a)+3(b, a, a) a-2\left(b, a, a^{2}\right)+\left\{\left(b, a, a^{2}\right)+\left(b, a^{2}, a\right)+\left(a, b, a^{2}\right)\right. \\
& \left.+\left(a^{2}, b, a\right)+\left(a, a^{2}, b\right)+\left(a^{2}, a, b\right)\right\} .
\end{aligned}
$$

The expression in braces is zero by the linearized form of (4); the remainder is the conclusion of the lemma.

LEMMA 2. Let $R$ be a nonassociative ring satisfying (1) and (2). Then

$$
\begin{aligned}
12(b, x, x) a= & -3\left\{\left(b, a, x^{2}\right)+\left(b, x^{2}, a\right)\right\} \\
& +5\{(b, x, a x)+(b, a x, x)\} \\
& +\{(b, x, x a)+(b, x a, x\} \\
& -[a,(b, x, x)]-x,(b, a, x)]-[x,(b, x, a)] .
\end{aligned}
$$

Proof. Linearize Lemma 1 to obtain

$$
\begin{aligned}
0 \equiv & \bar{F}(b, a, x)=2\left(b, a, x^{2}\right)+2(b, x, a x)+2(b, x, x a) \\
& -[a,(b, x, x)]-[x,(b, a, x)]-[x,(b, x, a)] \\
& -4(b, x, x) a-4(b, a, x) x-4(b, x, a) x .
\end{aligned}
$$

The proof follows since

$$
\begin{aligned}
0= & \bar{F}(b, a, x)+4 \bar{D}(b, x, a, x)-4 \bar{D}(b, a, x, x) \\
& -\bar{D}(b, x, a, x)-\bar{D}(b, x, x, a)-\bar{D}(b, a, x, x)=-3\left(b, a, x^{2}\right)-3\left(b, x^{2}, a\right) \\
& +5(b, x, a x)+5(b, a x, x)+(b, x, x a)+(b, x a, x)-[a,(b, x, x)] \\
& -[x,(b, a, x)]-[x,(b, x, a)]-12(b, x, x) a .
\end{aligned}
$$

The remainder of this section will deal with GRA rings.

LEMMA 3. If $R$ is a GRA ring, then for each fixed $b \in R, P_{b}=$ the additive subgroup spanned by $\{(b, x, x) \mid x \in R\}$ is a right ideal of $R$.

The proof is immediate from Lemma 2 and $0=\bar{C}(a, b, x)$. 
Lemma 4. If $R$ is a GRA ring, then

$$
(a, x, x)(b, y, y)+(a, y, y)(b, x, x)=0
$$

for all $a, b, x, y \in R$.

Proof. We call a map $D: R \rightarrow R$ a derivation if $(a b) D=$ $a(b D)+(a D) b$. From (1) it is clear that $a D_{x}=(a, x, x)$ is a derivation on $R$. From Lemma 3, $a D_{x, y}=((a, x, x), y, y)$ is also a derivation on $R$ since $((a, x, x), y, y)=((a, x, x) y) y-(a, x, x) y^{2} \in P_{a}$. This means

$$
(a b) D_{x, y}=a\left(b D_{x, y}\right)+\left(a D_{x, y}\right) b .
$$

In contrast to this, if we expand differently,

$$
\begin{gathered}
(a b) D_{x, y}=\left((a b) D_{x}\right) D_{y}=\left(\left(a D_{x}\right) b+a\left(b D_{x}\right)\right) D_{y}=\left(a D_{x} D_{y}\right) b \\
\left.+\left(a D_{x}\right)\left(b D_{y}\right)+\left(a D_{y}\right)\left(b D_{x}\right)+a\left(b D_{x} D_{y}\right)=\left(a D_{x, y}\right) b+\left(a D_{x}\right) b D_{y}\right) \\
+\left(a D_{y}\right)\left(b D_{x}\right)+a\left(b D_{x, y}\right) .
\end{gathered}
$$

Comparing this with the previous sentence gives $\left(a D_{x}\right)\left(b D_{y}\right)+$ $\left(a D_{y}\right)\left(b D_{x}\right)=0$; this is the identity of Lemma 4 .

LEMMA 5. If $R$ is a GRA ring, then $(a, x, x)(b, y, y)+(b, x, x)(a, y, y)=$ 0 .

Proof. $\quad(a, x, x)(a, y, y)=-(a, x, x)\{(y, a, y)+(y, y, a)\} \quad$ by (4) = $\{(a, a, y)+(a, y, a)\}(y, x, x)$ by Lemma $4=-(y, a, a)(y, x, x)$ by (4). We have established $(a, x, x)(a, y, y)=-(y, a, a)(y, x, x)$. Iterating this three times gives $(a, x, x)(a, y, y)=-(y, a, a)(y, x, x)=(x, y, y)(x, a, a)=$ $-(a, x, x)(a, y, y)$. Thus $(a, x, x)(a, y, y)=0$. We then linearize this in the element $a$ to get the identity of Lemma 5 . for $R$.

TheOREM 1. Let $R$ be a GRA ring. The following properties hold

(a) $I$ is an ideal of $R$.

(b) Every element of $I$ is a sum of elements of $\cup_{a \in R} P_{a}$.

(c) $[I, I]=0$.

(d) $\left(P_{a}\right)^{2}=0$ for all $a \in R$.

(e) $\left[I^{2}, R\right]=0$.

Proof. Property (b) is clear. Since $P_{a}$ is a right ideal for each $a \in R, I$ is also a right ideal; equation (1) then shows that $I$ is a two-ideal 
of $R$. This shows (a). We now show (c): $(a, y, y)(b, x, x)=$ - $(a, x, x)(b, y, y)$ from Lemma $4 \quad I=(b, x, x)(a, y, y)$ from Lemma 5. Lemma 5 also shows (d). We now show (e): from (2) we have $\left[a^{2}, a\right]=0$; linearizing this gives $\left[a^{2}, b\right]+[a b+b a, a]=0$. Now, if $i$ and $j$ are elements of $I$, and $b$ is an element of $R,[i j+j i, b]+[i b+b i, j]+$ $[j b+b j, i]=0$. From parts (a) and (c) we have $2[i j, b]=0$; therefore $\left[I^{2}, R\right]=0$.

Theorem 1 shows that $I$ is commutative. It follows that for each $a \in R, P_{a}$ is an ideal of the subring $I$. It also follows that $I$ is nil. Actually, we have shown $I$ is a Baer-lower-radical ring. We will go on and show a much stronger condition on nilpotence, but we will state the above result as a theorem now.

THEOREM 2. (a) If $R$ is a simple GRA ring, then $R$ is right alternative.

(b) If $R$ is a nil-semi-simple GRA ring, then $R$ is right alternative.

Lemma 6. Let $R$ be a GRA ring. Then

(a) $(a,(b, c, c), d)=(a, c, c) d \cdot b-(a, c, c) b \cdot d$.

(b) $(a,(b, c, c), b)=0$.

(c) $I[R, I]=[R, I] I=0$.

Proof of (a). By (1) and Lemma 3, the map $x D=(x, c, c) d$ is a derivation. Thus $(a b) D=(a D) b+a(b D)$. As in Lemma $4, D_{c}$ is also a derivation; so $(a b) D_{c}=\left(a D_{c}\right) b+a\left(b D_{c}\right)$. Combining these two expressions gives

$$
(a b, c, c) d=(a, c, c) d \cdot b+a \cdot(b, c, c) d=(a, c, c) b \cdot d+a(b, c, c) \cdot d .
$$

Therefore $(a,(b, c, c), d)=(a, c, c) d \cdot b-(a, c, c) b \cdot d$.

The statement (b) follows from (a). We now prove (c). Let $d \in I$. Then $(a,(b, c, c), d)=(a, c, c) d \cdot b-(a, c, c) b \cdot d \quad$ by $\quad$ (a) $=$ $(d,(a, c, c), b)$ by parts (a) and (c) of Theorem $1=-(d,(b, c, c), a)$ by part (b) of this proof. Now, continuing, $0=(a,(b, c, c), d)+(d,(b, c, c), a)=$ $a(b, c, c) \cdot d-a \cdot(b, c, c) d+d(b, c, c) \cdot a-d \cdot(b, c, c) a=d[a,(b, c, c)] \quad$ by parts (a), (c), and (e) of Theorem 1.

Since $d \in I$, we have shown that $I[R, I]=0$. By part (c) of Theorem 1 , we have $[R, I] I=0$ as well. This finishes the proof of Lemma 6.

LEMMA 7. Let $R$ be a GRA ring. Then,

(a) $K=\{([R, I], x, x) \mid x \in R\}$ is an ideal and $I K=K I=0$. 
(b) $A(I)=\{a \in I \mid(a, x, x)+a I+I a \subseteq K$ for all $x \in R\}$ is an ideal, $I \cdot A(I)+A(I) \cdot I \subseteq K$, and $[R, I] \subseteq A(I)$.

Proof of (a). $K$ is understood to be the subgroup spanned by the indicated elements. $\quad K$ is a right ideal of $R$ from Lemma 3. From (1) and Lemma 6.c, $b([R, I], x, x) \subseteq([b,[R, I]], x, x)+([R, I], x, x) b \subseteq$ $K$. Thus $K$ is a left ideal of $R$. By (1) and Theorem 1.c, if $i \in I$, $([r, i], x, x)=[r,(i, x, x)]$. Thus $I K+K I \subseteq I[R, I]+[R, I] I=0 \quad$ by Lemma 6.c.

Proof of (b). First notice that $A(I)$ is an ideal. This requires (1), part (a), Lemma 6.c, and Theorem 1.c. Clearly, $I \cdot A(I)+A(I) \cdot I \subseteq$ $K$. Lemma 6.c says $[R, I] \subseteq A(I)$.

THEOREM 3. Let $\langle(a, b, b)\rangle$ be the ideal of $R$ generated by the single associator $(a, b, b)$. Then $\langle(a, b, b)\rangle^{3}=0$.

Proof. By Lemma 3 and Lemma $7,\langle(a, b, b)\rangle \subseteq P_{a}+A(I)$. Using Theorem 1.d, $\langle(a, b, b)\rangle^{2} \subseteq P_{a} P_{a}+I \cdot A(I)+A(I) \cdot I+A(I) A(I) \subseteq K$. Therefore, $\langle(a, b, b)\rangle^{3} \subseteq I K+K I=0$.

COROllary. If $R$ is a semi-prime GRA ring, then $R$ is right alternative.

Proof. From Lemma 7.a, $K^{2}=0 \Rightarrow K=0$. From the proof of Theorem $3,\langle(a, b, b)\rangle^{2} \subseteq K=0$; thus $\langle(a, b, b)\rangle=0$. Since $(a, b, b)=0$ for all $a, b \in R, R$ is right alternative.

Example of a GRA ring. If $A$ is an associative and commutative ring with an element $1 / 2$, and $M$ is any module over $A$, then $\mathrm{S}=\mathrm{A} \times \mathrm{M}$ can be made into a GRA ring by the following definition of addition and multiplication. Addition is coordinatewise. Multiplication is given by $(a, m)\left(a^{\prime}, m^{\prime}\right)=\left(a a^{\prime}, 1 / 2 a m^{\prime}+1 / 2 a^{\prime} m\right)$. If we identify $M$ with $\{0\} \times M$, then $M$ is a two-sided ideal of $S$, and $[S, M]=0$. If $1 / 2+1 / 2=e(e$ is the identity of $A)$, then $e$ is an idempotent of $S$, and $-4(m, e, e)=m$ for all $m \in M$. The ring $S$ gives us a counterexample to various questions we might raise. For example:

1. There exist GRA rings which are not right alternative.

2. In a GRA ring $R, I$ need not be in the nucleus.

3. In a GRA ring $R,((R, x, x), x, x)$ need not be zero.

4. Based on the example $S$, one might attempt to show that in any GRA ring $R,[R, I]=0$. This we have not been able to show, but we have shown that for any element $b \in R,\langle((R, b, b), b, b)\rangle$ is an ideal of $R$ 
which commutes elementwise with $R$. This corresponds closely to $M$ since $M=\langle((S, e, e), e, e)\rangle$.

Related work. Hypotheses similar to (1), (2), and (3) have been studied. In [6] and [3], the three identities listed below were assumed.

(a) $(a b, c, d)+(a, b,[c, d])=a(b, c, d)+(a, c, d) b$.

(b) $(a, b, c d)+([a, b], c, d)=c(a, b, d)+(a, b, c) d$.

(c) $(a, a, a)=0$.

In [4], the condition (b) was replaced by flexibility;

(b') $(a, b, a)=0$ for all $a, b \in R$.

LEMMA 8. (Kleinfeld). If $R$ is a ring satisfying $(a),(b)$, and $(c)$, or $(a),\left(b^{\prime}\right)$, and $(c)$, then $R$ is a GRA ring.

Proof. We must show $([b, a], a, a)=0$ for all $a, b \in R$. From (a) and (c) we have $([b, a], a, a)=-[a,(b, a, a)]$. The proof of Lemma (1) required only (a) and (c). Therefore,

$$
\begin{aligned}
0 & =[a,(b, a, a)]+4(b, a, a) a-2\left(b, a, a^{2}\right) \\
& =3([b, a], a, a)-2\left\{\left(b, a, a^{2}\right)+([b, a], a, a)-a(b, a, a)-(b, a, a) a\right\}
\end{aligned}
$$

If (b) holds, the expression in braces is 0 , and hence $([b, a], a, a)=0$. If $\left(\mathrm{b}^{\prime}\right)$ holds, using (a) and $\left(\mathrm{b}^{\prime}\right)$, we get $\left(b, a, a^{2}\right)-([b, a], a, a)-a(b, a, a)-$ $(b, a, a) a=0$. As above, we get

$$
0=-([b, a], a, a)-2\left\{\left(b, a, a^{2}\right)-([b, a], a, a)-a(b, a, a)-(b, a, a) a\right\} .
$$

Therefore, (a), (b'), and (c) imply (3).

THEOREM 4. If $R$ is a semi-prime ring satisfying (a), (b), and (c), or $(a),\left(b^{\prime}\right)$, and $(c)$, then $R$ is alternative.

Proof. By the corollary to Theorem $3, R$ is right alternative. If (b') holds, then $R$ is alternative. If (b) holds, by the mirror form of the corollary to Theorem $3, R$ is left alternative. Thus $R$ is alternative in this case as well.

Theorem 4 is an impressive generalization of [3], [4], [5], and [6].

I. R. Hentzel and G. M. Piacentini have studied rings satisfying only conditions (1) and (2). They have shown that when such rings are simple 
and possess an idempotent, then they must be right alternative. In view of this result, it seems that perhaps equation (3) is not necessary. This seems even more plausible since only equations (1) and (2) imply the result

$$
([[a, b], c], c, c) \equiv 0 \text {. }
$$

The proof of Lemma 3 requires that $([a, x], x, x)=0$ for all $a, x$ in the ring. Without this, $D_{a, b}$ is not a derivation. The fact that $D_{a, b}$ was a derivation was the basis of all our results.

\section{REFERENCES}

1. I. R. Hentzel, Characteristic in non-associative ring. to appear.

2. I. R. Hentzel and G. M. Piacentini, Generalization of right alternative rings, to appear, Trans. Amer. Math. Soc.

3. E. Kleinfeld, Generalization of alternative rings, I. J. Algebra, 18 (1971), 304-325.

4. - Generalization of alternative rings. II. J. Algebra, 18 (1971), 326-339.

5. —, A generalization of $(-1,1)$ rings, Pacific J. Math., $53 \cdot(1974), 195-202$.

6. H. F. Smith, Prime generalized alternative rings with non-trivial idempotent, Proc. Amer. Math. Soc., 39 (1973), 242-246.

Received May 17, 1974. This paper was written while the author held an Iowa State University Science and Humanities Research Institute grant.

IOWA STATE UNIVERSITY 


\section{PACIFIC JOURNAL OF MATHEMATICS}

\section{EDITORS}

RICHARD ARENS (Managing Editor)

University of California

Los Angeles, California 90024

\author{
R. A. Beaumont \\ University of Washington \\ Seattle, Washington 98105
}

\section{J. DugundII}

Department of Mathematics

University of Southern California

Los Angeles, California 90007

D. Gilbarg and J. Milgram

Stanford University

Stanford, California 94305

\section{ASSOCIATE EDITORS}
E. F. BECKENBACH
B. H. NeumanN
F. WoLF
K. YoshidA

\section{SUPPORTING INSTITUTIONS}

\author{
UNIVERSITY OF BRITISH COLUMBIA \\ CALIFORNIA INSTITUTE OF TECHNOLOGY \\ UNIVERSITY OF CALIFORNIA \\ MONTANA STATE UNIVERSITY \\ UNIVERSITY OF NEVADA \\ NEW MEXICO STATE UNIVERSITY \\ OREGON STATE UNIVERSITY \\ UNIVERSITY OF OREGON \\ OSAKA UNIVERSITY
}

\author{
UNIVERSITY OF SOUTHERN CALIFORNIA \\ STANFORD UNIVERSITY \\ UNIVERSITY OF TOKYO \\ UNIVERSITY OF UTAH \\ WASHINGTON STATE UNIVERSITY \\ UNIVERSITY OF WASHINGTON \\ AMERICAN MATHEMATICAL SOCIETY
}

The Supporting Institutions listed above contribute to the cost of publication of this Journal, but they are not owners or publishers and have no responsibility for its contents or policies.

Mathematical papers intended for publication in the Pacific Journal of Mathematics should be in typed form or offset-reproduced (not dittoed), double spaced with large margins. Underline Greek letters in red, German in green, and script in blue. The first paragraph or two must be capable of being used separately as a synopsis of the entire paper. Items of the bibliography should not be cited there unless absolutely necessary, in which case they must be identified by author and Journal, rather than by item number. Manuscripts, in duplicate, may be sent to any one of the four editors. Please classify according to the scheme of Math. Reviews, Index to Vol. 39. All other communications should be addressed to the managing editor, or Elaine Barth, University of California, Los Angeles, California, 90024.

100 reprints are provided free for each article, only if page charges have been substantially paid. Additional copies may be obtained at cost in multiples of 50.

The Pacific Journal of Mathematics is issued monthly as of January 1966. Regular subscription rate: $\$ 72.00$ a year (6 Vols., 12 issues). Special rate: $\$ 36.00$ a year to individual members of supporting institutions.

Subscriptions, orders for back numbers, and changes of address should be sent to Pacific Journal of Mathematics, 103 Highland Boulevard, Berkeley, California, 94708.

PUBLISHED BY PACIFIC JOURNAL OF MATHEMATICS, A NON-PROFIT CORPORATION Printed at Jerusalem Academic Press, POB 2390, Jerusalem, Israel.

\section{Copyright (C) 1975 Pacific Journal of Mathematics All Rights Reserved}




\section{Pacific Journal of Mathematics

Vol. 60, No. $2 \quad$ October, 1975

Waleed A. Al-Salam and A. Verma, A fractional Leibniz q-formula ........... 1

Robert A. Bekes, Algebraically irreducible representations of $L_{1}(G) \ldots \ldots \ldots \ldots 11$

Thomas Theodore Bowman, Construction functors for topological

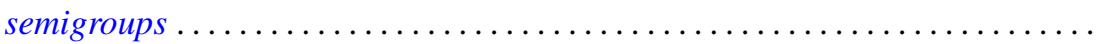

Stephen LaVern Campbell, Operator-valued inner functions analytic on the

closed disc. II .........................................

Leonard Eliezer Dor and Edward Wilfred Odell, Jr., Monotone bases in $L_{p} \ldots \ldots$.

Yukiyoshi Ebihara, Mitsuhiro Nakao and Tokumori Nanbu, On the existence of

global classical solution of initial-boundary value problem for

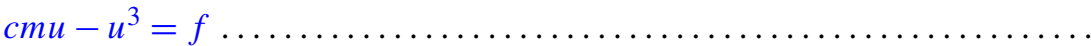

Y. Gordon, Unconditional Schauder decompositions of normed ideals of

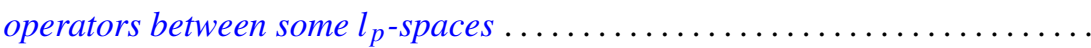

Gary Grefsrud, Oscillatory properties of solutions of certain nth order functional

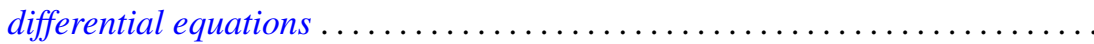

Irvin Roy Hentzel, Generalized right alternative rings ...................

Zensiro Goseki and Thomas Benny Rushing, Embeddings of shape classes of compacta in the trivial range .................................

Emil Grosswald, Brownian motion and sets of multiplicity . .

Donald LaTorre, A construction of the idempotent-separating congruences on a

bisimple orthodox semigroup .

Pjek-Hwee Lee, On subrings of rings with involution ...

Marvin David Marcus and H. Minc, On two theorems of Frobenius ...

Michael Douglas Miller, On the lattice of normal subgroups of a direct

product. .

Grattan Patrick Murphy, A metric basis characterization of Euclidean space

Roy Martin Rakestraw, A representation theorem for real convex functions ....

Louis Jackson Ratliff, Jr., On Rees localities and $H_{i}$-local rings ...

Simeon Reich, Fixed point iterations of nonexpansive mapping . .

Domenico Rosa, $B$-complete and $B_{r}$-complete topological algebras ...

Walter Roth, Uniform approximation by elements of a cone of real-valued

functions ....

Helmut R. Salzmann, Homogene kompakte projektive Ebenen

Jerrold Norman Siegel, On a space between $B H$ and $B_{\infty} \ldots$

235

Robert C. Sine, On local uniform mean convergence for Markov operators

James D. Stafney, Set approximation by lemniscates and the spectrum of an

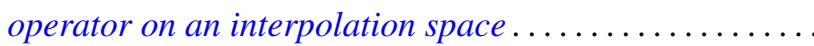

Árpád Száz, Convolution multipliers and distributions .......

Kalathoor Varadarajan, Span and stably trivial bundles ..........

Robert Breckenridge Warfield, Jr., Countably generated modules over

commutative Artinian rings....................... 